\title{
How Does Time Flow in Living Systems? Retrocausal Scaffolding and E-series Time
}

\author{
Naoki Nomura ${ }^{1} \cdot$ Koichiro Matsuno ${ }^{2} \cdot$ Tomoaki Muranaka $^{3} \cdot$ Jun Tomita ${ }^{4}$
}

Received: 13 September 2018 / Accepted: 7 July 2019 / Published online: 22 July 2019

(C) The Author(s) 2019

\begin{abstract}
Anticipatory acts or predictive behavior are prerequisites for living organisms to sustain their survival when escaping from a predator, catching prey, or schooling. For example, catching prey requires that the predator perform some procedures that are equivalent to estimating the directional movement of the prey, its speed and its distance relative to the predator. Underlying these procedures is time experience, which does not adhere to man-made mechanical clocks. Living organisms keep time based on the local activities of each participant and form ecological clocks together. The timekeeping of ecological clocks has been called E-series time, which is interactive in character and consists of mutual alignment of timing that is co-adjusted to each other's movements and rhythms. A main objective of our current work is to illustrate how E-series time is used for flows of anticipatory acts. To explain such predictive moves and their efforts based on how the perspective of the immediate future affects the present, we resort to the organismic activity of revising the preceding acts in retrospect and semiotic scaffolding that extends beyond simple linear causality. Special attention is paid to the construction of the notion of retrocausal scaffolding, which is a series of dialogical punctuations or mutual coordination of rhythms for the joint production of the present moment of now. Retrocausal scaffolding is synonymous with negotiated anticipation, which is a semiotic/communicative account of revising the preceding acts in the present moment.
\end{abstract}

Keywords Punctuation $\cdot$ Semiotic scaffolding $\cdot$ Predictive models $\cdot$ Nonlinear causality $\cdot$ Eseries time $\cdot$ Moment signs $\cdot$ Anacrusis

\section{Introduction}

For any form of life, from unicellular organisms to large-brained mammals, living in a predictable environment is essential for increasing its chances for survival. We all need to know what to expect in the next few moments to improve our prospects of survival;

Naoki Nomura

nomura@hum.nagoya-cu.ac.jp

Extended author information available on the last page of the article 
otherwise, smooth locomotion and movement may not be possible. Imagine that an old man walks out suddenly and crosses in front of you. You avoid colliding with him by quickly calculating the distance and time needed to avoid him. This calculation is a prediction, and this prediction requires a time frame that is not separate from space. Such a time frame is distinctly different from our ordinary clock's time frame, which does not take space into account.

Prediction entails a time factor, and accurate high-speed time assessment is imperative to achieving one's goals, such as avoiding a collision or pursuing a prey. Therefore, without reliable timekeeping and time assessment, prediction is not possible. Furthermore, this time assessment is spatially bound, which leads to the notion that predictive moves require different types of spatially bound "clocks" other than our ordinary clocks. What type of time measuring is necessary? Reliable time measurement in biology cannot be based on our ordinary clocks, because living organisms do not use our standard time. In our view, biological time measurements are based on the local activities of every organism in its communication environment, which generates time ecologically. Thus, organisms adopt what we call the time code in the E-series for foraging, fighting, escaping, or mating (Nomura et al. 2018; Nomura and Matsuno 2016). Quick predictions of a few moments ahead in the near future are essential for living beings to sustain their lives. Thus, looking forward is the key for survival. In this paper, we argue that quick predictive moves in interaction constitute such ecological time flows.

We humans often recognize two types of time progressions, or "time arrows". One type is the standard time based on our ordinary clocks. For example, we make an appointment to meet and gather at a certain place on a scheduled time and day. Thus, standard time represents the time that is usually referenced. The other type is our personal time, which is colored by our own experience and may or may not correspond to the standard time. This time is often psychological, such as your experience of time lag resulting from a long flight or of time slowing down while listening to a boring lecture. While the former type is said to be objective and unidirectional, the latter may be more subjective and filled with individual memories, long-term anticipation, etc.

However, neither of these types of time progression suits the flow of time directly applicable to living organisms in action because the time frame that living organisms use to predict the near future is neither objective nor subjective. Seasonal migrations of birds and whales, appearances of cicadas every 13 years, and the circadian rhythms of organisms entrained in day-night cycles are all interactions with the environment and other neighboring participants. These organisms measure time neither mechanically nor individually but rather ecologically. The regular movements of the sun and moon during the day and night and seasonal or annual changes are all signs by which living organisms keep time and decide subsequent actions. Biological time is neither objective nor subjective but rather is inter-subjective based on communication with other individuals and its environment. We explain how this third type of time, E-series time, flows in the biological world.

\section{Time as Punctuation}

To begin with, what is time? Time is difficult for us to fathom and articulate. The practical yet enigmatic nature of time represents a profound theme for humans in everyday life, religion, philosophy, politics, art, and science (Aristotle 2015; Augustine 1961; Heidegger 
1996; Ricoeur 1984; Takiura 1976). In biology, time and temporality are also relevant topics in terms of inner clocks, such as circadian rhythms, of memories and tense, and of biological evolution (Pittendrigh 1958; Uexküll and Kriszat 1957; Fernández 2010; Kull 1998; Cowley 2015; Fraser 2007; Kawade 2006; Matsuno 2016; Tomita et al. 2005; Muranaka and Oyama 2016). Putting aside the ontological aspects of time or momentarily suspending the question of whether something called time really exists or not, an alternative viable method of viewing time may be through signs and their activities or meaning-making efforts during the communication process (Nomura et al. 2018; Nomura and Matsuno 2016).

The known processes of time are all recognized by some type of distinction. The ticking of the hands of a clock, the bells of a church, and fallen leaves in autumn indicate the progression of time with some type of distinction having been noticed by (or communicated with) an organism. Thus, the agential reading of timekeeping necessitates some type of boundary or punctuation, without which organisms cannot recognize the passage of time. Each step of a punctuation, which is "a difference that makes a difference" (Bateson 1972), indicates a boundary between events that have already occurred and those that are going to occur next (Nomura et al. 2018). Such steps may represent the nature of temporality in the biological world. The distinction made by a punctuation is the smallest meaning-making unit of information and can be described as semiotic activity producing a difference that counts. We believe that a promising method for conceptualizing time is to view it as a meaning-making system shared by the participating subjects or organisms. For us, time is a sign activity, and if a distinction is not perceived, then time has not occurred. This statement, however, presents another perspective: if time is represented by such distinctions, then a different mode of punctuation would make up a different time. Although already discussed elsewhere (Nomura et al. 2018), a schematic summary of time as a meaning-making system is attached (Table 1) to help guide further discussion.

An ordinary clock tells us that objective time moves forward with global synchrony, meaning that your watch and mine synchronize without mutual negotiations by virtue of the radio control or the declaration of standard time (ST). The mode of ticking (i.e.,

Table 1 A summary of all of the introduced time series. The matrix shows the four constituents of time as a meaning-making system: (1) grammar or mode of punctuation; (2) code that indicates the character of a clock; (3) method of timekeeping; and (4) identity of the timekeeper (Based on Nomura et al. 2018)

Time as a meaning making system

\begin{tabular}{|c|c|c|c|c|}
\hline Time & A-series time & $\begin{array}{l}\text { B-series } \\
\text { time }\end{array}$ & $\mathrm{C}$-series time & E-series time \\
\hline $\begin{array}{l}\text { Grammar } \\
\quad \text { (Mode of } \\
\text { punctuation) }\end{array}$ & $\begin{array}{l}\text { tense } \\
\text { (past-present-- } \\
\text { future) }\end{array}$ & $\begin{array}{l}\text { sequence } \\
\quad \text { (earlier/later) }\end{array}$ & array & co-adjusting \\
\hline Code (Clock) & $\begin{array}{l}\text { subjective, } \\
\text { internalized } \\
\text { individual code }\end{array}$ & $\begin{array}{l}\text { objective, externalized } \\
\text { global code }\end{array}$ & $\begin{array}{l}\text { static, non-active } \\
\text { code }\end{array}$ & $\begin{array}{l}\text { synchroactive } \\
\text { Relational code }\end{array}$ \\
\hline $\begin{array}{l}\text { Method of } \\
\text { timekeeping }\end{array}$ & $\begin{array}{l}\text { by memory and } \\
\text { anticipation }\end{array}$ & by global-synchrony & no timekeeping & $\begin{array}{l}\text { by local- } \\
\text { synchronization }\end{array}$ \\
\hline Timekeeper & $\begin{array}{l}\text { the first-person } \\
\text { agency }\end{array}$ & $\begin{array}{l}\text { the third-person } \\
\text { observer }\end{array}$ & no timekeeper & $\begin{array}{l}\text { the second-person } \\
\text { negotiators }\end{array}$ \\
\hline
\end{tabular}


punctuation) is universal everywhere; thus, international appointments can be made. We call such objective time B-series time (McTaggart 1908, 1927; Nomura et al. 2018). B-series time represents a code name given to the Newtonian notion of lineally progressive time. On the other hand, personally sensed subjective time under the influence of individual experiences, such as jet-lag or time slowing down, moving fast or being frozen, is called A-series time (McTaggart 1908, 1927; Nomura et al. 2018). Here, the basis of a time series is one's sense of the past, present and future, i.e., tense, where boundary making depends on the individual's pace and tempo. Each of these two times corresponds to objective time and subjective time, and in this paper, they are both treated as semiotic codes instead of natural objects.

Now, the reader of this article may ask: Is A-series time applicable to living organisms other than humans? Our answer would be "yes," and living organisms as agents seem to have their own subjective sense of the past, present, and future, at least in limited scopes. When anteaters capture ants one by one, they should have their own subjective time that distinguishes the immediate past (definiteness in retrospect) and the immediate future (indefiniteness in prospect). Receptor time, as explained by Uexküll, is defined as the duration of a human's single moment, which is estimated as approximately $1 / 18$ of a second (Uexküll and Kriszat 1957). Motion pictures thus utilize this tempo and project 18 pictures a second to imitate natural movement. Fighting fish have approximately 50 moments a second, whereas vineyard snails are said to have less than 4 moments a second (Uexküll and Kriszat 1957, p. 340-341). The reason moments as an indivisible time unit differ among organisms is that these sensations are the species' own signs expressing a moment (i.e., moment signs), which may correspond to our notion of punctuation. Subjects who live in different Umwelt thus differ in their expression of time sensations and receptor time. Fighting fish, which feed on fast-moving prey, have species-specific advantages that make the prey's movement appear to occur in slow motion (Uexküll and Kriszat 1957: p. 340-341). Similarly, jazz musicians (as a sort of "species") can perceive quick-moving harmonic twists as moment signs. The classic jazz standard Moment's Notice (Coltrane 1958) may be a good example. Time is a semiotic act and not a thing; therefore, when time is viewed from the individual's perspective based on his/her/its own past, present and future, time appears to be in the A-series.

\section{E-Series Time}

E-series time is interactive in its character and distinctly different from the objective Bseries or the subjective A-series, both of which have neither spatial nor self-vs-other (i.e., dialogical) dimensions. The term E-series time originated because McTaggart (1927) used "D-series time" as an offshoot of C-series time; thus, the next series available was E-series time. E-series time is composed of two or more interacting agents or organisms jointly punctuating toward a mutual alignment of their movement (i.e., rhythm alignment or moment signs exchanges) (Nomura et al. 2018; Nomura and Matsuno 2016). Imagine a case of a pair dancing or schooling fish whose activities require coordinated step by step movements or maintenance of swimming proximity. Thus, the type of time used and kept here entails spatial and self-other dimensions. In both cases, ongoing communication between the participating organisms and even between their sub-component systems also is necessary for co-adjustment to each other's movements and rhythms. 
E-series time is a local act of synchronization using a relational time code that is neither individual nor global. This local time is ubiquitous and is observed in many areas, such as in human conversations, walking side by side, chorus singing, predatory cooperation among lions, migratory birds flying in V-formations, and mosquitoes swarming in the air. Obviously, standard clock time cannot be used. Because timekeeping in this series occurs using multiple agencies in a dialogue-like situation, the present moment of now is co-adjusted by negotiating each other's receptor time.

A man and his guide dog each possess species-specific moment signs and thus must adjust their moment of now to realize smoothly coordinated walking movements together (Uexküll and Kriszat 1957) and conform to a trajectory that attempts to produce their collaborative now. The grammar of this time series is therefore the coadjusted temporal spans in rhythms and movements, i.e., punctuations. Such interrelated participants have been called second-person negotiators, because co-adjustments of their movement are interactively determined within the negotiable range for the participants (Nomura et al. 2018). This type of time spanning in the E-series is radically different from the tense-less ST, which is stringently set and observable by the thirdperson observer (in the B-series), and is also different from the individual and subjective tempo activated through one's past, present, and future by the first-person agency (in the A-series), which does not assume the dimension of negotiations.

Physicists have determined the length of one second in an exact manner, and our ordinary clocks punctuate every second to tell us the current global time. Punctuation applied to reading the standard clock is simply a matter of physicist conventions, which is how objective time is kept. Importantly, we do not participate in time making. On the other hand, distant memories are placed in our individual time frame and create distinctions when making a boundary of the recalled event, such as "the picnic" or "the ceremony", which explains how subjective time is kept without interference from the outside. In both cases, however, punctuation (or boundary making) is the basis of time awareness.

Compared with the present moment established globally by ST, the E-series time observed in living systems is unique because it fabricates the present moment of now from within. A progressive state of joint action from one moment to the next is required, and the participants are the timekeepers for each other in this interactive context. In Eseries time, punctuation is jointly performed via constant interactions among two or more participants or organisms, who coordinate each other's receptor time or temporal spanning. This time is inter-subjective in the sense that the participating agents are making mutual "tick marks" in an effort to hit the mark exactly, although they continually fail to hit the mark, leading to further trial and updating. Complete synchronization or perfect agreement on time intervals, if maintained, deprives the interaction of its momentum. A pair of dancers or a flock of birds continually challenges the impossible and adjusts their error movements in an effort to align their movements, stay in rhythm and keep up with each other or with the neighboring birds.

\section{Prey Interception with Internal Models: Dragonfly and Mosquito}

Prey interception is a behavior that is common to many insects. The predator's interception course cannot be pre-planned, because the prey will make unexpected turns. Thus, the predator insect's steering control has been considered purely reactive. 
However, a study of dragonflies indicated that these insects relied on both predictive and reactive control (Mischiati et al. 2015). Imagine a dragonfly chasing a mosquito and catching it in the air. According to these scientists, dragonflies predict the movements of their prey by second-guessing where the prey will be in the near future. Such anticipation of the flight path of the prey has not been demonstrated among insects previously. A dragonfly's interception of a mosquito is guided by not only the target's changing position but also the dragonfly's ability to turn its body and align itself with the mosquito's flight path (See Fig. 1). The dragonfly catches the prey from beneath and behind, which reduces the risk of detection. Such a standardized alignment across flights suggests that a specific body orientation is preferred for grasping the prey.

Both the dragonfly's body turns and body alignment before the attack indicate that the insect has a future image of its attack. This behavior may be similar to that of a tennis player who knows how the ball should be hit in the near future and thus aligns his/her body and positions the racket before hitting the ball. Scientists consider this alignment to be based on "internal models" (Franklin and Wolpert 2011; Zago et al. 2004; Flanagan et al. 2001; Kawato 1999; Wolpert et al. 1995) for prediction of the movement of prey. However, it should be noted that an internal model itself has no time factor and is similar to a map or a guide to be used as a scenario. This scheme is timeless, and this type of timeless time code belongs to C-series time (McTaggart 1927; Nomura et al. 2018).

For instance, the face of a clock, a monthly calendar, timetables, and music scores are in the $\mathrm{C}$-series, which is related to time that remains static and inactive by itself. When viewed as a picture, such time-related lists, drawings or compositions belong to the $\mathrm{C}$-series. $\mathrm{C}$ series time is a series of punctuated boundaries that form an array and do not specify the direction of movement. The composed products, such as a calendar or timetable, remain non-temporal (or static by themselves) despite the presumed work of the composer or author. That is, these products represent a map of time without the user. For example, a map of terrain can be used to climb a mountain or to descend it, and the direction is up to the user and can go either way. Before being used or interpreted, the map remains only a drawing.

The user of a C-series time map is the semiotic agent or organism that can decide the direction of movement or interpret how to use the map as a scenario, a text, or an internal model. An orchestra conductor with a musical score frozen in the C-series time code participates in semiotic interplay with the orchestra players during the actual performance.

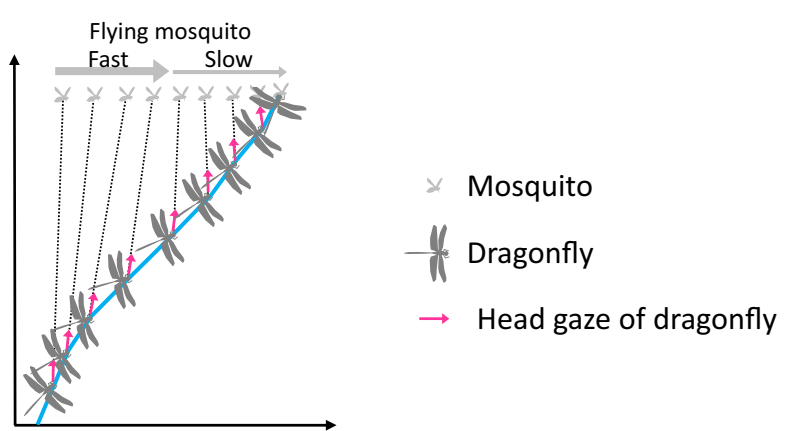

Fig. 1 A dragonfly's head and body orientation during prey interception: fixed gaze on the target while aligning its body for the future attack (Mischiati et al. 2015) 
The circadian rhythms of plants are generated in gene circuits in cells composed of multiple feedback loops. Such feedback loops by themselves can be considered a C-series time map.

In fact, the complicated coordination of the muscle cells of the dragonfly for flight locomotion has already been coded in complex signaling pathways with the use of participating neurotransmitters, such as acetylcholine. That is, the code frozen in C-series time is actually an already completed semiotic product that can be read by the organism repeatedly if required. Behavioral plasticity can accompany the rigidity of the biochemical code.

Dragonflies lock on to their target, visually track the predicted movements, and prepare for reactive responses to any unexpected movement that strays from the expected trajectory. Thus, the predator has an image of the prey over the next few moments and intercepts the target.

How are the flying speed and direction of the dragonfly in the pursuit adapted to the changes in the mosquito's position? Again, this situation is similar to that of an old man suddenly walking out in front of you and your avoidance of the collision. Distance and speed together must be taken into account to catch the prey or avoid the collision. Time and space are all one in the operation; that is, they are intact. Thus, the time code used for this purpose can neither be individualized nor internalized (in the A-series), which does not assume interactions, nor global nor definitive (in the B-series), which does not assume space. The time code must be interactive and inter-subjective (in the E-series) to accompany the moment-to-moment changes of position in space (Table 1).

A pursuing agent and an escaping agent constitute a complementary communicative system in which both engage in meaning-making: catching and escaping (Bateson 1979: p. 213). This system is constituted through the cyclic operation of mutual feedback and may be said to possess clock characteristics assuming the appropriate timing for responses. The dragonfly visually locks onto the target to track the predicted movement of the mosquito. To coordinate its action and speed, the dragonfly anticipates its action in advance, thereby acting from the perspective of the immediate future to affect the present.

Now, we wish to discuss the semiotic dynamics that permits mutual alignment of receptor time or moment sign exchanges, which roughly correlate to how E-series time is generated and flows. To prepare for the argument, we believe that two key ideas, reverse causation and semiotic scaffolding, are essential for its groundwork. At this point, this reverse causation occurs exclusively when revising the preceding act later in retrospect and by no means implies making the effect precede the cause. If an organism's temporal operation is a semiotic process, then we must expound on the precise nature of the process. If quick predictive movements of living organisms are the result of internal models, then the internal models of future scenarios affect the current action as if the future affects the present; reverse causation may become relevant in such cases. Furthermore, if semiotic scaffolding is roughly equivalent to an organism's communication pattern utilized by the organism itself as suggested by Hoffmeyer (2015), then time maneuvers must be included in that scaffolding process.

\section{Reverse Causation and Nonlinear Causality}

We ordinarily think that cause must precede effect and that this relationship owes itself to linear time progression that can stand by itself. Time is said to run from 
the past to the future via the present or from earlier to later and not vice versa. This firm conviction was the crux of classical science before the advent of cybernetics and information theory, and it is still widely shared. However, if an organism has a future goal in mind (i.e., prediction), then the expected future state becomes the cause of the present behavior, such as hurrying to catch a commuter train. Or, the dragonfly's future goal precipitates changes in its body alignment prior to an attack on its prey. Here, the direction of causality is reversed and called reverse causation or retrocausality. If causality is associated with linearly progressive time (of the B-series), then a logical dilemma or a paradox may occur in which time apparently goes backward from the future to the present in reverse causation.

When matter and energy are featured in physical systems, linear causality takes precedence, such as in the case of thermodynamic entropy, in which cooled gas does not heat up without intervention. The unidirectional time of the B-series remains applicable. When communication and control are the problem, information processing leads to foresight in that the cyclic operation between one's goal and the subsequent action connected through feedback (i.e., feedback loops) plays a key role in changing a cause to an effect and vice versa in circular fashion (Matsuno 2019). Such behavior is frequently observed in conversational quarrels among humans. A decisive question at this point is how loops appear in this material world. Such cyclic violation of linear causality, in which a future projection affects current actions and states, is widely recognized under the rubric of nonlinear causality.

Note here that linear causal relationships are subsumed under the cyclic relationship, as observed in transformations such as $\mathrm{J} \rightarrow \mathrm{K} \rightarrow \mathrm{L} \rightarrow \mathrm{M} \rightarrow \mathrm{N}$ and back to $\rightarrow \mathrm{J}$, in which the adjacent transformation $\mathrm{J} \rightarrow \mathrm{K}$ is forwardly causal and the roundabout transformation $\mathrm{K} \rightarrow \mathrm{J}$ is reversely causal. The citric acid cycle represents an example. Separating the two types of causation is only superficial, and linear causation makes sense only when a sequential segment of the entire reaction cycle is cut into parts, such as $\mathrm{L} \rightarrow \mathrm{M}$ or $\mathrm{M} \rightarrow \mathrm{N}$. From the circular standpoint, admitting global adherence of linear causality to linearly progressive time is not necessary, since both linear and circular causalities are involved in the same system. Thus, the terms feedforward or feedback can be considered different aspects of the same cyclic transformation.

Furthermore, identifying the cause in a linear fashion becomes troublesome in this circular causation because the cause of $\mathrm{K}$ can be reduced to $\mathrm{J}$ as well as to $\mathrm{L}, \mathrm{M}, \mathrm{N}$, or even to $\mathrm{K}$ itself. Restricting a cause to a single code is problematic in terms of nonlinear causation, because identifying a cause is an active movement of an arbitrary choice, such as doggedly searching for a single cause of worldwide economic recession. In other words, although causes have a type of metaphysically abstract nature, effects are always concrete-particular in their implications, which refer to an action that has already been completed experientially.

Thus, reverse causation (i.e., retrocausality) is not peculiar with regard to predictive behavior in terms of information, coding, and control. In this way, reverse causation is absorbed into circular causation, and a paradox appears only when linear causality is tied to linearly progressive B-series time. In the circular paradigm, the terms feedforward and feedback together constitute cyclic transformations. 


\section{Reverse Causation: Acting for the Present in the Immediate Future}

Having expressed our position on reverse causality, we now proceed to exemplify biological cases. If near-future anticipation prompts a dragonfly to intercept its target, then the future-input (prediction) affects the present-output (action), which contrasts with cases such as the observation coming first and triggering the outset of the action. Such a case is similar to a tennis player who knows how a coming ball should be hit and is guided by internal models to assume a particular hitting position before the ball arrives. These situations are, we think, examples of acting for the present in the immediate future, and such situations are ubiquitous.

The prey pursuit of a dragonfly necessitates anticipatory actions, because the dragonfly constantly corrects and adjusts its flying relative to the flight of a mosquito. Each movement by the dragonfly during the pursuit is the result of a reactively active response to changes observed in the behavior of the prey. These movements are dialogical in the sense that the dragonfly predicts and reacts based on differences in the movement of the mosquito and corrects its position; subsequently, the mosquito further adjusts its own position to escape by observing the new position of the predator. A gazelle chased by a lion may present similar behavior. For the dragonfly, the mosquito is an environmental variable that is anticipatory and the object of interaction. The result can be seen as turn taking based on anticipatory movements, which continues until the chase ends.

One of the essential rules for dialogical exchange is turn taking (Sacks et al. 1974) between participants. Each participant has its own expectations and predictions. In conversation, it is customary for one speaker at a time to participate in an alternating manner. Simultaneous utterances by two or more participants make it difficult to sustain conversation. In another example, Tinbergen's well-known illustration of the spawning steps of sticklebacks is made possible by keeping the goals of the male and female fish in mind as they alternate their actions (Tinbergen 1953). Once a certain stage in the trajectory is reached, an early error, if any occurs, does not count in the future steps.

Therefore, to achieve successive turn taking, participants must act afterward to correct an error, where the action in the present in effect nullifies or disqualifies a past move. A mispronunciation in conversation may be corrected or "repaired" soon afterward so that the error is disqualified. A dragonfly's updated current position in prey pursuit can ignore the previous positions, meaning that they no longer count. This action can also be taken as reverse causality because it is a correction of the past event in the present. However, this reverse-causal account does not imply that the past is changed in the present. When a predator holds a near-future image of the prey and takes anticipatory actions toward it, the actions can be seen as a correction of the present event in the future.

Thus, in the biological sphere, both feedforward and feedback work together under dialogical paradigms or nonlinear causalities. Errors lead to answers in both cases, indicating reverse causation, where the sequence of reactive activity is significantly restrained compared to the actions that could potentially be selected. A dragonfly's error-actuated body alignment in prey pursuit restrains its behavior within a certain range in catching the prey, such as from beneath and behind. The use of certain words in conversation narrows the speaker's choice of the next utterance to fewer options, which in turn makes the following speech more predictable for the participants. Other viable options that are not selected remain in the background. Error detection occurs as a result of feedback and restrains future options. 
Imagine two athletes running side by side and following a preceding vehicle driven by a coach. Figure 2 roughly indicates the changes in distance between the runners (blue and green) and the lead car. Five meters (vertically shown by the orange line) is the approximate distance between the athletes and the vehicle. The set parameter of a 5 $\mathrm{m}$ interval controls or restrains the behavior of the two runners, although the moving condition of the vehicle itself might be affected by traffic. The 5-m distance to the lead vehicle is a predictive model, a tentative scenario of which the athletes must be aware. Each running position is part of the whole system constituted by/with the other runner's state as well as the lead car's forward movement.

The approximate distance to the vehicle is the near-future image (predictive model) of each runner, and the runners execute their strides one after another, acting in the immediate future in order to revise the present. Each act may be an act of anticipation. However, the deviations from the ideal interval, such as a-2 and b-2, are nullified and disqualified by subsequent acts, and they remain only in the record. The present choice of action can easily disqualify past errors, which may represent the principle of trialand-error in the cybernetic sense.

These characteristics indicate an occurrence of reverse causality rather than causality, because the set interval as a scenario for the future affects the current mode of action. The deviations that have been made before in time, such as a-2 and b-2, are "repaired" by the subsequent act at a-3 and b-3 as if the deviations did not occur as errors.

\section{Semiotic Scaffolding: A Schematic View}

The concept of scaffolding is well known in psychology. This concept was originally proposed by the Russian developmental psychologist Lev Vygotsky (1986) and represents the distance between the actual developmental level of a child and their potential developmental level (See Fig. 3). However, Vygotsky never used the term scaffolding. His famous theory, the zone of proximal development (ZPD), indicates the transitional learning area between what a child can do alone and what a child cannot do without the help of others. The overlapping area between the two stages indicates the developmentally undetermined area, in which a child may acquire knowledge or skill through appropriate support from the teacher. The two areas located before and after the ZPD are the area of learning already completed and that of learning yet to be completed. The crossover in-between indicates the area of learning that can be accomplished with guidance and encouragement, which is the proximal zone where actual learning occurs.

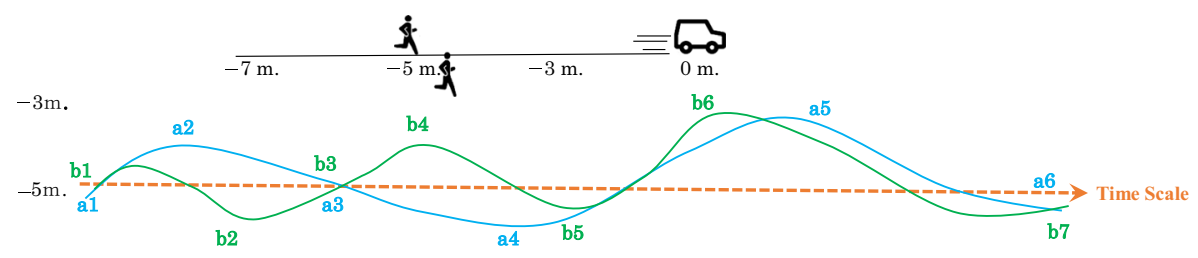

$-7 \mathrm{~m}$.

Fig. 2 Changes in the interval between the lead vehicle and two runners: The orange horizontal line is the time scale, which indicates (in the vertical direction) a 5-m distance to the lead vehicle. The letters (blue a and green b) show the distance of each to the vehicle at a given point in time 
Vygotsky's zone of proximal development (ZPD)

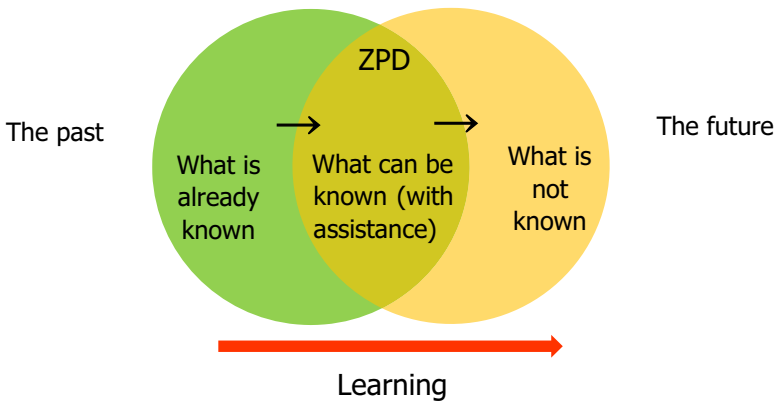

Fig. 3 Vygotsky's zone of proximal development (ZPD)

The idea of the ZPD was picked up and later developed by psychologists J. Bruner and others under the name "scaffolding." Through this scaffolding, a child obtains new skills and knowledge by being offered "support points" or "stepping stones" (Wood et al. 1976). The concept was further interpreted by J. Hoffmeyer for the biological sciences under the name semiotic scaffolding, which represents a web of checks and balances established through communication between organisms (i.e., a historically created interaction mechanism) (Hoffmeyer 2015, p. 154). A concrete example is revising the preceding act at a later moment. Emmeche (2015) rephrases Hoffmeyer's point by stating that semiotic scaffolding guides a system's behavior to follow a more definite sequence of events, indicating the network of semiotic controls. Favareau (2015) uses a framework of cybernetic jargon to show that the definite sequence of events from semiotic scaffolding is a type of interactional constraint and possibility bias.

Let us explain our view regarding time progression schematically based on semiotic scaffolding. For this purpose, a situation should be presented in which there is no semiotic scaffolding, which is similar to an artist's white canvas before drawing. However, we also will use the following two examples throughout for illustration purposes: one is a parent teaching a child how to ride a bicycle, and the other is a bird teaching a young bird to fly. Thus, Fig. $4 \mathrm{a}$ is an undefined space that includes only a frame. That is, there is no punctuation and hence "no difference that makes a difference," and only the arena (time and space) of activity is provided, such as the day/ season and environment for the riding or flying. In the jargon of the ZPD, only the zones, the areas focused on learning, are granted. Here, an action that occurs inside the space has no way of being evaluated due to the absence of criteria. There is no difference; thus, the action does not lead to any time.

Figure $4 \mathrm{~b}$ provides vertical and horizontal lines that form a matrix. The lines can be considered pre-scaffolding for an organism to act upon the space and make interpretations. Only with the presence of agency can the matrix function as scaffolding; however, there is no agency, and no rule has been established for utilizing the matrix at this stage. Greater degrees of freedom are observed in this figure; thus, our action can be started from any place downward from the upper right corner or radially from the central space. The zones in the ZPD, such as the Known, Can be Known, and Not Known (Fig. 3), may correspond to this stage, in which no learning direction is specified. This stage is 
(a)

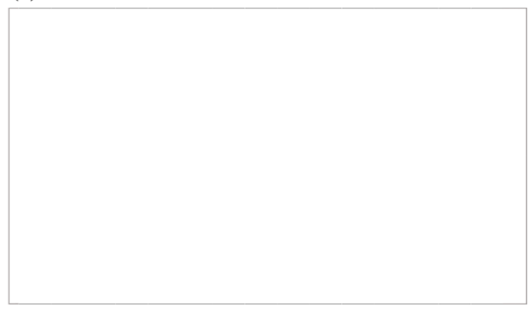

(c)

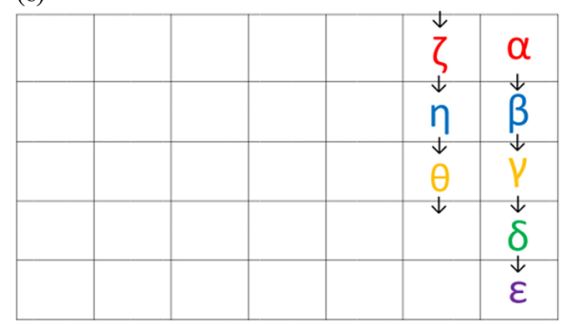

(b)

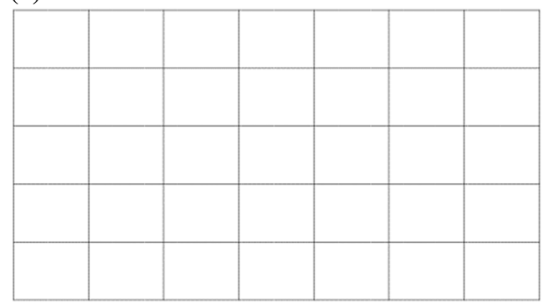

Fig. 4 a Blank space: a schematic view of no semiotic scaffolding except for the frame or the universe of discourse. (e.g., a white canvas before being drawn on by an artist or an animal's initial encounters before the first gambit). b Space with a matrix: a schematic view of semiotic scaffolding. $\mathbf{c}$ Space used according to a rule: a schematic view of semiotic scaffolding. d Space used for a calendar: a schematic view of semiotic scaffolding

similar to the stage for checking the bicycle as a vehicle beforehand or for the mother bird stopping to feed her young. For both riding and flying, they still remain a set-up or static scheme that needs a rule to make it workable. Unless acted upon, the scheme is simply a picture corresponding to the $\mathrm{C}$-series, and Fig. $4 \mathrm{~b}$ remains in a pre-scenario (See also Table 1). Such a scheme is similar to a clock's face without the two hands.

The next step is to introduce a rule onto the matrix regarding use. If rules are set, then the lines in the space become scaffolding that can "guide a system's behavior to follow a more definite sequence of events" (Emmeche 2015: p. 275). If a child occupies a box in the upper right corner and continues to occupy boxes vertically one by one, then their actions will determine the direction of movement. For example, the Greek alphabet is inscribed vertically as follows: $\rightarrow$ (See Fig. 4c). Once the rule for progression has become clear, the writing can act as a sort of primitive clock because it has a series of progressive punctuations; however, what type of time would it keep? We do not know at this point. This stage also corresponds to the ZPD, in which the parent briefly holds the child's bicycle to assist the first and second pedal strokes, parent ducks actually push their ducklings out of the nest to force them to use their wings, and parent eagles fly with their young together to illustrate the process of catching the wind in the air. Definite stepping stones exist in the above scenarios.

Next, the matrix can be used to approach a time indicator (i.e., a calendar), in which the rule becomes clearer because each box counts a single day and the days move horizontally from left to right (See Fig. 4d). If the timekeeper is a bystander-observer or the third-person observer (e.g., someone looking at next year's calendar), the scheme appears to him/her to be in unidirectional time, indicating before/after relationships. 
Such time becomes the self-running B-series time. B-series time corresponds to linearly progressive clock time or the ST in our civilization, in which everyone agrees on how to read the punctuation following the suggestions of physicists. This time is objective in nature and indicates only earlier/later or before/after relations. B-series time shows a sequence but remains tense-less; therefore, it does not tell us anything about the past, present, or future. The B-series clock is globally synchronized and externalized because it is read by all of the concerned bystanders, the third-person observers, who do not participate in its making. The ingenuity of B-series time leads to the inevitable selection of the tense-less time code whenever the space-time coordinate is introduced.

If, however, the timekeeper is a first-person agent (or someone actually using this calendar) positioning June 18th in one of the unit boxes that indicates "today," the scheme appears to her to be an ongoing continuation from the past to the future via the present. Such time becomes self-narrating A-series time, for which she can put a check mark on her own decision. A-series time has been defined as subjective time assuming a single agent has access to its own memory and anticipation (Nomura et al. 2018). The essence of A-series time is centered around tense, or a sense of duration or an unbroken succession of events that constitute one's own past, present, and future. For example, we know that the timing of saying "good night," which indicates an individual's end of the day, may not occur at midnight but instead is a choice of the individual. A-series time seems to presume agential capacity in that the transformation of tense is made possible by a single experiencing subject coordinating different tenses (See Table 1).

However, if you and I are in a communicative relationship, neither you nor I alone can make a unilateral decision over the definition of a day's passage. Deciding the beginning and end of the day becomes a matter of working out or negotiation because we are secondperson negotiators working toward collaborative decisions. In this case, turn taking of punctuation and mutual time alignments are necessary to co-fabricate the present moment of now or today from within. Such locally synchronized time corresponds to E-series time, figuratively speaking. The second-person negotiators as the participants (i.e., internal observers) have a vision of the scenario and anticipate near-future punctuations in advance and in response to each other. Therefore, June 18th is jointly checked based on the shared knowledge that June 19th lies ahead and can be expected, as is the case for athletes guided by the lead car at 5-m intervals. Thus, the matrix (Fig. 4d) can be the semiotic scaffolding or predictive model that assists us in negotiating and deciding the beginning and ending of the day and in providing a scheme for future joint action. This assisting guidance may also represent a reverse causality because the scheme that extends to the future prompts us to define the current position (Matsuno 2016, 2017). E-series time control permits one to foresee the very next step and possesses the $\mathrm{C}$-series scheme as its prediction model. Both the novice bicycle rider and the young flying eagle have attained their own schemes (i.e., prediction models) for riding or flying, which will be an interaction with the parent's or mother eagle's schemes and adjustments of their temporal spans to achieve joint actions.

\section{Retrocausal Scaffolding}

We have discussed reverse causation (i.e., retrocausality) and semiotic scaffolding. The notion of reverse-causal scaffolding or retrocausal scaffolding comes from these two subjects. Reverse causality has been referred to as quick predictive movements derived 
from the future image that is held by an organism and permits its readiness for action (i.e., anticipatory behavior). The dragonfly's prey pursuit has been used as an example. Semiotic scaffolding corresponds to the network of communication for predictive moves that guides and constrains the actions to follow. The utterances in conversation that constrain the scope of possible replies of the other speaker has been used as an example.

Thus, we define retrocausal scaffolding as a series of dialogical punctuation and mutual coordination of rhythms (i.e., temporal spanning) that allow future actions to affect the present. The term retrocausal scaffolding is adopted to designate such a series of support points and stepping stones as punctuation for the joint production of now. Although semiotic scaffolding provides insights into the static side of support systems, such as a "web of checks and balances" (Hoffmeyer 2015: p. 154) or "network of semiotic control" (Emmeche 2015: p. 275-289), retrocausal scaffolding focuses on the continuous revision of predictive models resulting from interactive pushing and pulling. Self-corrective anticipatory acts are executed and updated in a dialogical setting by such second-person negotiators. Retrocausal scaffolding is thus synonymous with negotiated anticipation because the anticipatory system interacts with a system that is itself anticipatory. Living organisms use such semiotic operations permitted by the Eseries time code in their quick predictive movements.

Thus, negotiated anticipation is not a psychological/individual concept for a single organism but rather an interactive/dynamic concept between organisms that describes moment-to-moment changes in prediction and execution. Individuals engaged in patterned conversational exchanges or rhythmic musical performances, such as in a calland-response, feel greater confidence in estimating the next action of a partner due to the projected potential embedded in the exchange. This scenario can also be expressed as an instance of carrying out retrocausal cohesion (Matsuno 2019), which explains the emergence of feedback loops. Although Rosen's “closure to efficient causation" (Rosen 1991) was originally formally conceived on an abstract level, the catalysts needed for its operation must be generated internally (Cárdenas et al. 2010). Incorporating the catalysts into the closure is also an integral part of retrocausal cohesion.

Of course, at the same time we must be ready to react to any unexpected deviation from the initial estimate, such as in the dragonfly's reactive control during prey pursuit. A biological illustration may help explain retrocausal scaffolding more concretely (See Fig. 5: Among the three existing versions of the video clip, the version with natural sounds of the caterpillar eating a leaf is used instead of the silent version or the version with typewriter sounds).

The caterpillar is the larva of a butterfly, and it eats a leaf in rhythmic movements and continues to eat parts of the leaf in the shape of an arc. The arc-shaped pattern is further grazed to create a new arc. This action is repetitious, and we recognize that the caterpillar expects and is ready for the next round of movement. This readiness is seen both at the end of a single grazing movement and at the start of the return movement, because the caterpillar can gather momentum accordingly. We are familiar with this type of action, aren't we? For example, in the phrase "Ready!", "Go!", "ready" is understood as a momentum-building term, and in the phrase "One, two, three!" when taking a photograph, the terms "one" and "two" are not necessary for the actual taking of the photo but may be necessary for the subjects to match their breathing and synchronize with each other in a joint action. 


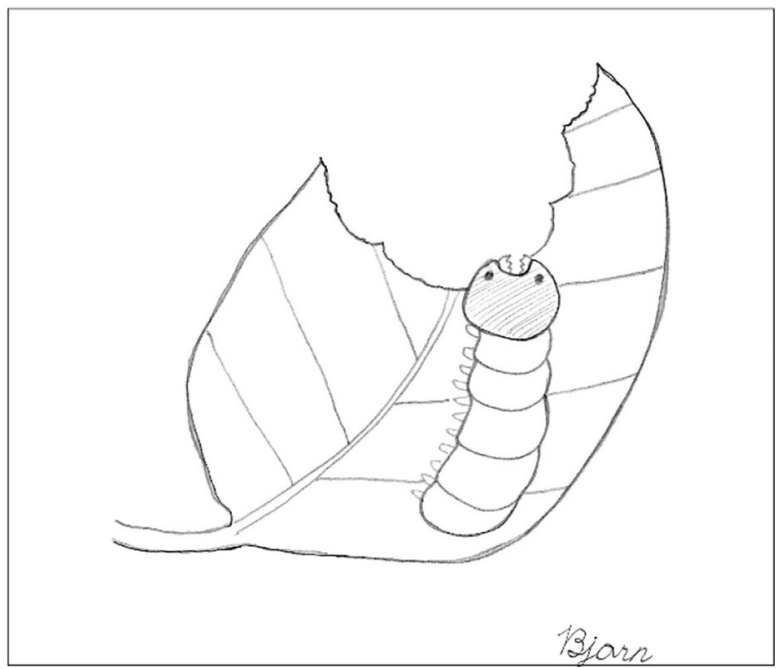

Fig. 5 A caterpillar (i.e., larva) of a tiger swallowtail butterfly eating a leaf. Permission obtained from ViralHog https://www.youtube.com/watch?v=7k4w7nwIQAs

This phenomenon in music is called anacrusis, which means a set of notes or beats preceding the legitimate part of the musical phrase. It is an upbeat that consists of the unaccented notes before the first downbeat in a bar, which is often called a pickup. In the case of "Ready!", "Go!", "ready" corresponds to the "upbeat," or anacrusis. That is, the anacrusis is a lead-in or a push-up for the subsequent downbeat. Therefore, an anacrusis is anticipatory and directed toward a future event (Hasty 1997: p. 120-129). The caterpillar shows anacrusis by expecting its future event, which is the next arc of biting.

The parallel between the caterpillar's swift predictive moves and anacrusis in music seems obvious. Although parallelism is observed in music theory, in biology a reversal may be observed in which anacrusis is one of the basic systems by which living organisms learn to acquire their time frame and to synchronize themselves with their own environment. That is, anacrusis is a cohesive factor for pulling in something to come. The musical concept of anacrusis is perhaps derived from the predictive movements shared widely among living organisms. The point is, however, that the projective potential of anacrusis is anticipatory (Butterfield 2006; Hasty 1997), and anacrusis corresponds to retrocausal scaffolding, which is aimed at future events.

Let us consider why a caterpillar eating a leaf can be explained in terms of the E-series with second-person negotiators instead of the A-series with first-person agents acting upon the object (Matsuno 2018). Of course, it is not wrong to view the caterpillar's biting as a single anticipatory agent acting upon a non-anticipatory object (Rosen 1985; Louie 2010); however, we propose an alternative view of the same phenomenon from an interactional/communicative standpoint. When organisms act toward an object, the organism and the object together constitute a feedback loop in which differences (i.e., information) are transmitted via cybernetic circuitry. Consider a blind man with a stick walking on the street. To understand the blind man's locomotion or behavior, the man, the stick and the street must be all taken into account, since the stick is the pathway along which the transformations of difference are being transmitted (Bateson 1972: p. 465). The recursive transmitting process involving the street, the man and the stick goes 
indefinitely round and round. Similarly, the caterpillar and the leaf together should represent a method for delineating the system; therefore, we do not eliminate any of these relevant pathways of communication. This situation does not mean that the leaf possesses the same intentional capacity as the caterpillar, although it does mean that the change in shape of an eaten leaf transmits a meaningful difference to the caterpillar and thus affects its next bite. The changing shape of the leaf and the caterpillar's eating behavior become synchronized rather than simply are synchronized, which is similar to the movement of the stick being "in sync" with the walking of the blind man. The term second-person negotiators is thus a metaphor for such a recursive network of control through feedback and feedforward. The relationship between an organism and its subjective environment (i.e., Umwelt) may be similar, with both aspects constituting the communication network required to produce synchronicity or shared temporality.

\section{How Retrocausal Scaffolding Generates the Now}

We have argued that predictive moves displayed by living organisms are based on retrocausal scaffolding, which is negotiated anticipation in E-series time. Then, how does retrocausal scaffolding generate E-series time (or the succession of now) across different biological cases? We tentatively offer the following three working explanations of how retrocausal scaffolding helps bring about E-series time: (1) by constructing predictive models, (2) by making the system robust, and (3) by canceling out errors.

(1) Constructing predictive models: Retrocausal scaffolding may help construct a predictive model or an internal model. The dragonfly's image of its future attack on a mosquito, the caterpillar's anticipatory anacrusis by building momentum for the next arc of biting, and a tennis player's preformation of his/her posture before actual ball hitting all seem to indicate the existence of internal models that help these agents act in the future to affect the present.

In our view of time, the prediction model associated with anticipatory acts is a type of prescenario or a static scheme before actualization that corresponds to the C-series time code of the non-active clock. However, we should be careful that such an internal model is not an absolutely fixed program. The model of any concerned agent must be adaptable to respond to environmental changes. If the prey is a fruit fly instead of a mosquito, then the dragonfly's pursuit may be adapted accordingly; if the caterpillar eats harder leaves, its rhythmic pattern of biting and anacrusis are affected; if a tennis player adjusts his/her posture depending on the opponent's hitting style, then the hit is affected, etc.

These examples suggest frequent changes or rewriting of internal models, whose text is inscribed in the $\mathrm{C}$-series time code. Thus, as in a music score for jazz improvisers, living organisms' internal models are not a fixed schedule but a working draft that always remains ready for further editing.

There may be two ways of editing and rewriting the pretext, which is the internal predictive models or the C-series time code. In "first-order editing," minor changes within plasticity may involve changes in the state (Ashby 1956), such as the dragonfly adjusting its flying speed for each mosquito, the caterpillar making different arc shapes 
for each leaf, or the tennis player controlling his/her swing speed. For the outside observer, the C-series time code looks complete and static; however, for the internal participants, it is a working draft or a pretext that is ready to be edited and activated, and it is a design that can fluctuate. The participants make use of the local degrees of freedom (or the scope of possible changes within plastic boundaries) for their minor behavioral adjustments.

In contrast, "second-order editing" represents changes in the first-order changes and thus is higher in abstraction. These acts involve major and grammatical changes (in quality) expressed as changes in the way of behaving (Ashby 1956). Examples are the dragonfly chasing entirely different insects instead of mosquitoes, the caterpillar eating different species of leaves, or the tennis player transforming his/her whole style of play. Since these are changes in the change of the state, second-order editing is considered higher in abstraction than the former examples, which results in major revisions of the $\mathrm{C}$-series time code and suggests substantial changes in local perspective. In the case of a dragonfly switching its target from a mosquito to a bee, the dragonfly switches from one local perspective to another, more dependable $\mathrm{C}$-series time code for its new semiotic scaffolding. The previous grammar may be abandoned because of a failure in the resource intake, and such discarding might have evolutionary implications.

Whether the editing is minor or major, retrocausal scaffolding mobilizes intersubjective time so that the static proto vision of internal models can be transferred to instantaneous predictive moves in action.

(2) Making the system robust: Robustness is not a given attribute but something interactively created through time under the dynamics of retrocausal scaffolding. We know that interaction in a dialogical setting brings about a patterned stylized exchange in which "pattern" is an abstraction that affects behavior similar to a predictive model. What seems critical is the time factor. Patterns can be described as static pictures, although in communicative reality, they are time dependent in the sense that only the continuous creation of now can hold its pattern. Without acting in the future to affect the present or without resorting to the E-series time code, patterned communication cannot be realized and maintained because it requires mutual fit and synchronization. Note also that the fit is the result of continuous exclusion of the unfit.

Anacrusis of the caterpillar increases the difficulty of selecting other movements as alternatives. If the biting is repeated and becomes recursive, it creates a mode or a pattern, which makes the series of action robust by dismissing other potentially selectable movements. The robustness of the caterpillar's action is obtained at the cost of trimming away potential alternatives.

Robustness can also be observed at the molecular level. The citric acid cycle that produces ATP in mitochondria has 7 main steps of molecular changes that form a loop: citrate $\rightarrow$ isocitrate $\rightarrow \alpha$-ketoglutarate $\rightarrow$ succinate $\rightarrow$ fumarate $\rightarrow$ malate $\rightarrow$ oxaloacetate and back to citrate. What is noticeable is the chemical affinity between the incoming carbon elements from the outside and the remaining ones in the cycle that carry the path-dependent histories (memories) behind themselves (Matsuno 2015). The cyclic operation of the citric acid cycle is made possible by the chemical affinity that excludes non-affined elements. The robustness of the system is obtained by shutting out other possible alternatives. 
(3) Canceling out errors: Retrocausal scaffolding cancels out previous errors in the trial. Each step of the biting of a leaf by the caterpillar is constant trial-and-error. If an error occurs, it is corrected afterward because correction may be permitted. The corrective action in the present sets aside the past errors as if they did not exist. The caterpillar's eating is continually updated so that the previous arc-shaped bite marks are no longer there and do not account for its moment of now.

Although the two runners keeping pace with the lead vehicle often deviate from the set distance, the deviations are cleared by their return to the $5-\mathrm{m}$ interval. The former deviations or errors are canceled out in the present moment, and they remain in the record only in the B-series time code. Thus, the present choice of action can disqualify past errors in the recursive feedback loop run by the E-series.

To achieve successive turn taking in conversation or joint action, the participants must act to correct their errors afterward when the action in the present in effect nullifies or invalidates past moves. Otherwise, the ongoing processes are difficult to maintain. This action is also reverse causal because it is a correction of the past event in the present. Conversation analysts call it "repair," meaning correcting an utterance immediately after making it (Moerman 1988).

\section{Final Comments}

We started this paper with a simple fact that prediction is imperative for any organisms to live and that predictive movements must assume a time factor. This time factor, however, is not the one derived from standard linear time (i.e., the B-series), which does not take space into account, and it is likely space-intact time or ecological time, which has been called E-series time. Whether it is subjective, objective, inter-subjective or otherwise, time recognition is based on some type of boundary making or punctuation. Whatever the time series is, including ST, the constant updating of punctuation generates the sequence of time. Without such demarcations, timekeeping is not possible from the semiotic/ communicative perspective (Nomura et al. 2018; Nomura and Matsuno 2016).

The goal of the present paper has been to elucidate the semiotic process of how Eseries time is generated and how it flows in predictive biological circumstances. Nonetheless, we should give the reader due warning that the use of the term "flow" does not imply movement of an object, such as that of water, but rather is a metaphoric expression for ongoing semiotic operations. Thus, A-series time kept by an individual's own tempo can "flow" from the past to the present and from the present to the future for an agent that can assume its continuous being or identity, whereas B-series time determined by an external global clock "flows" from the earlier to the later in equal time intervals according to uniform linear motion. C-series time, such as predictive models, is defined as static and does not "flow." E-series time "flows" when interactive efforts are made locally toward generating mutual synchronization between the internal participant-observers. All these time series are expressions that occur through semiotic processes (See Table 1).

To illustrate how E-series time is generated, we detoured around the problems of reverse causation and semiotic scaffolding to attain the new concept of retrocausal scaffolding, which we believe can explicate the semiotic dynamics of E-series time. 
Our detour clarifies the following points: (1) retrocausality can be separated from the linear progression of time; that is, reverse causation is not always dependent on the reverse direction of time because of nonlinear causality; (2) although semiotic scaffolding originates in the ZPD and has spatial tones (e.g., zone, web, or network), the concept has been extended to cover temporality, as shown in the time sequences that appear through the matrix using the notion of punctuations (Figs. 4a-d); and (3) internal/predictive models that organisms possess for anticipatory behavior have a static character or an image of time in stasis, although the translation of such models to one of the time codes bridges the psychological concept (i.e., internal models) and the language of time ( $\mathrm{C}$-series time). Further, the $\mathrm{C}$-series time code is defined based on movement, and it is subject to minor and major revisions under the varying interactive pressures of its environment.

Based on these concepts, we are able to explain how the concatenation of now with now - the linking of which is equated to E-series time - is not contradictory but instead is theoretically sound. One reason is that anticipatory behavior with a predictive model is reverse causal and at the same time is independent from linear progressive time. Punctuations are made to ensure that the interactive system returns to the permissible range of the negotiated now, where neither tense nor sequence but instead the concurrent now is time's essence. The agency for the concatenation of now with now has recourse to the cohesive capacity unique to now for acting on the present based on the perspective of the immediate future. The reader may be reminded of the caterpillar utilizing anacrusis or an "upbeat" to rhythmically move on to the next bite that is yet to come. We have called such micro-timing by organisms, or the reverse-causal alignment of temporal spanning, acting for the present in the immediate future. The negotiation of the moment signs between interacting organisms can occur with the projected potential of their internal models. Organisms and E-series time must not be separated for both to be alive.

Acknowledgements We thank ViralHog for their kind generosity in permitting us to use the video clip shown in Movie 1.

\section{Compliance with Ethical Standards}

Conflict of Interest The authors declare that they have no conflicts of interest.

Open Access This article is distributed under the terms of the Creative Commons Attribution 4.0 International License (http://creativecommons.org/licenses/by/4.0/), which permits unrestricted use, distribution, and reproduction in any medium, provided you give appropriate credit to the original author(s) and the source, provide a link to the Creative Commons license, and indicate if changes were made.

\section{References}

Aristotle. (2015). Physics, Vol. IV. Translated by R.P. Hardie and R.K. Gaye. Adelaide: The University of Adelaide.

Ashby, W. R. (1956). An introduction to cybernetics. London: Methuen.

Augustine. (1961). Confessions. Translated by R. S. Pine-Coffin. Harmondsworth: Penguin Books.

Bateson, G. (1972). Steps to an ecology of mind. New York: Ballantine Books.

Bateson, G. (1979). Mind and nature: A necessary unity. New York: E. P. Dutton. 
Butterfield, M. W. (2006). The power of anacrusis: Engendered feeling in groove-based musics. MTO: A Journal of the Society for Music Theory., 12(4), 1-17.

Cárdenas, M. L., Letelier, J. C., Gutierrez, C., Cornish-Bowden, A., \& Soto-Andrade, J. (2010). Closure to efficient causation, computability and artificial life. Journal of Theoretical Biology, 7, 263(1), 79-92.

Coltrane, J. (1958). Moment's notice. In Blue Train (BLP 1577), Blue Note Records.

Cowley, S., \& Steffensen, S. (2015). Coordination in language: Temporality and time-ranging. Interaction Studies, 16(3), 474-494.

Emmeche, C. (2015). Semiotic scaffolding of the social self in reflexivity and friendship. Biosemiotics, 8 , 275-289.

Favareau, D. (2015). Symbols are grounded not in things, but in scaffolded relations and their semiotic constraints (or how the referential generality of symbol scaffolding grows minds). Biosemiotics, 8, 235255.

Fernández, E. (2010). Life and temporality: The place of biosemiotics within Peirce's general semiotics. Chinese Semiotic Studies, 4(2), 289-300.

Flanagan, J. R., King, S., Wolpert, D. M., \& Johansson, R. S. (2001). Sensorimotor prediction and memory in object manipulation. Canadian Journal of Experimental Psychology, 55, 87-95.

Franklin, D. W., \& Wolpert, D. M. (2011). Computational mechanisms of sensorimotor control. Neuron, 72, 425-442.

Fraser, J. T. (2007). Time and time again: Reports from a boundary of the universe. Leiden. Boston: Brill.

Hasty, C. (1997). Meter as rhythm. Oxford University Press.

Heidegger, M. (1996). Being and time. Translated by J. Stambaugh. New York: State University of New York Press.

Hoffmeyer, J. (2015). Introduction: Semiotic scaffolding. Biosemiotics, 8, 153-158.

Kawade, Y. (2006). Biosemiotics: Biology of subjectivity. Kyoto: Kyoto University Press Japanese.

Kawato, M. (1999). Internal models for motor control and trajectory planning. Current Opinion in Neurobiology, 9, 718-727.

Kull, K. (1998). Organism as a self-reading text: Anticipation and semiosis. International Journal of Computing Anticipatory Systems, 1, 93-104.

Louie, A. H. (2010). Robert Rosen's anticipatory systems. Foresight, 12(3), 18-29.

Matsuno, K. (2015). On the physics of the emergence of sensorimotor control in the absence of the brain. Progress in Biophysics \& Molecular biology, 119, 313-323. https://doi.org/10.1016/j. pbiomolbio.2015.08.004.

Matsuno, K. (2016). Retrocausality in quantum phenomena and chemical evolution. Information, 7, 62. https://doi.org/10.3390/info7040062.

Matsuno, K. (2017). From quantum measurement to biology via retrocausality. Progress in Biophysics \& Molecular biology, 131, 131-140.

Matsuno, K. (2018). Tempolarity naturalized. Philosophies, 3, 45. https://doi.org/10.3390 /philosophies3040045.

Matsuno, K. (2019). Retrocausal regulation for the onset of a reaction cycle. BioSystems, 177, 1-4. https://doi. org/10.1016/j.biosystems.2019.01.006.

McTaggart, J. E. (1908). The unreality of time. Mind; A Quarterly Review of Psychology and Philosophy., 17, $456-473$.

McTaggart, J. E. (1927). The nature of existence (Vol. II). Cambridge: Cambridge University Press.

Mischiati, M., Lin, H., Herold, P., Imler, E., Olberg, R., \& Leonardo, A. (2015). Internal models direct dragonfly interception steering. Nature, 517, 333-338.

Moerman, M. (1988). Talking culture: Ethnography and conversation analysis. Philadelphia: University of Pennsylvania Press.

Muranaka, T., \& Oyama, T. (2016). Heterogeneity of cellular circadian clocks in intact plants and its correction under light-dark cycles. Science Advances, 2(7), e1600500.

Nomura, N., \& Matsuno, K. (2016). Synchronicity as time: E-series time for living formations. Cybernetics and Human Knowing, 23(3), 69-77.

Nomura, N., Muranaka, T., Tomita, J., \& Matsuno, K. (2018). Time from semiosis: E-series time for living systems. Biosemiotics, 11(1), 65-83. https://doi.org/10.1007/s12304-018-9316-0.

Pittendrigh, C. S. (1958). Perspectives in the study of biological clocks. In A. A. Buzati-Traverso (Ed.), Perspectives in marine biology (pp. 239-268). Berkeley: University of California Press.

Ricoeur, P. (1984). Time and narrative vol. 1. Translated by K. Mclaughlin and D. Pellauer, Chicago: University of Chicago.

Rosen, R. (1985). Anticipatory systems: Philosophical, mathematical \& methodological foundations. Oxford: Pergamon Press. 
Rosen, R. (1991). Life itself: A comprehensive inquiry into the nature, origin, and fabrication of life. New York: Columbia University Press.

Sacks, H., Schegloff, E. A., \& Jefferson, G. (1974). A simplest systematics for the organization of turn-taking for conversation. Language, 50(4), 696-735.

Takiura, S. (1976). Time: A philosophical inquiry. Tokyo: Iwanami. (Japanese).

Tinbergen, N. (1953). Social behaviour in animals. London: Chapman and Hall.

Tomita, J., Nakajima, M., Kondo, T., \& Iwasaki, H. (2005). No transcription-translation feedback in circadian rhythm of KaiC phosphorylation. Science, 307, 251-254.

Uexküll, J., \& Kriszat, G. (1957). A stroll through the worlds of animals and men. In C. H. Schiller (Ed.), Instinctive behavior: The development of a modern concept (pp. 5-80). New York: International University Press.

Vygotsky, L. (1986). Thought and language (rev. Ed.) (A. Kozulin, Trans.). Cambridge: MIT Press. (Original work published 1934).

Wolpert, D. M., Ghahramani, Z., \& Jordan, M. I. (1995). An internal model for sensorimotor integration. Science, 269, 1880-1882.

Wood, D., Bruner, J., \& Ross, G. (1976). The role of tutoring in problem solving. Journal of Child Psychology and Psychiatry, 17, 89-100.

Zago, M., et al. (2004). Internal models of target motion: Expected dynamics overrides measured kinematics in timing manual interceptions. Journal of Neurophysiology, 91, 1620-1634.

Publisher's Note Springer Nature remains neutral with regard to jurisdictional claims in published maps and institutional affiliations.

\section{Affiliations}

\section{Naoki Nomura ${ }^{1} \cdot$ Koichiro Matsuno ${ }^{2} \cdot$ Tomoaki Muranaka $^{3} \cdot$ Jun Tomita ${ }^{4}$}

Koichiro Matsuno

CXQ02365@nifty.com

Tomoaki Muranaka

muranaka@ecology.kyoto-u.ac.jp

Jun Tomita

tomita@phar.nagoya-cu.ac.jp

1 Graduate School of Humanities and Social Sciences, Nagoya City University, Nagoya, Japan

2 Nagaoka University of Technology, Nagaoka, Japan

3 Center for Ecological Research, Kyoto University, Kyoto, Japan

4 Graduate School of Pharmaceutical Sciences, Nagoya City University, Nagoya, Japan 